\title{
Effectiveness of Health Education Teachers and School Nurses Teaching Sexually Transmitted Infections/Human Immunodeficiency Virus Prevention Knowledge and Skills in High School
}

Elaine A. Borawski, PhD ${ }^{a}$ Kimberly Adams Tufts, DNP, WhNP-BC, FAAN ${ }^{b}$ Erika S. Trapl, PhD ${ }^{c}$ Laura L. Hayman, PhD, RN, FAAN ${ }^{d}$ Laura D. Yoder, MPHe Loren D. Lovegreen, PhD $^{f}$

\section{ABSTRACT}

BACKGROUND: We examined the differential impact of a well-established human immunodeficiency virus (HIV)/sexually transmitted infections (STIs) curriculum, Be Proud! Be Responsible!, when taught by school nurses and health education classroom teachers within a high school curricula.

METHODS: Group-randomized intervention study of 1357 ninth and tenth grade students in 10 schools. Twenty-seven facilitators (6 nurses, 21 teachers) provided programming; nurse-led classrooms were randomly assigned.

RESULTS: Students taught by teachers were more likely to report their instructor to be prepared, comfortable with the material, and challenged them to think about their health than students taught by a school nurse. Both groups reported significant improvements in $\mathrm{HIV} / \mathrm{STI} /$ condom knowledge immediately following the intervention, compared to controls. Yet, those taught by school nurses reported significant and sustained changes (up to 12 months after intervention) in attitudes, beliefs, and efficacy, whereas those taught by health education teachers reported far fewer changes, with sustained improvement in condom knowledge only.

CONCLUSIONS: Both classroom teachers and school nurses are effective in conveying reproductive health information to high school students; however, teaching the technical (eg, condom use) and interpersonal (eg, negotiation) skills needed to reduce high-risk sexual behavior may require a unique set of skills and experiences that health education teachers may not typically have.

Keywords: reproductive health; sex education; STD and HIV education; intervention facilitator.

Citation: Borawski EA, Tufts KA, Trapl ES, Hayman LL, Yoder LD, Lovegreen LD. Effectiveness of health education teachers and school nurses teaching sexually transmitted infections/human immunodeficiency virus prevention knowledge and skills in high school. J Sch Health. 2015; 85: 189-196.

Received on July 3, 2013

Accepted on August 14, 2014

$\mathrm{H}$ gh rates of sexually transmitted infections (STIs) and human immunodeficiency virus (HIV) among adolescents have long been worrisome to healthcare professionals and health educators. In 2011, more than 1.7 million cases of chlamydia and gonorrhea were reported, with adolescent girls (ages 15-19) and minorities bearing a significant burden. ${ }^{1}$ Adolescents aged 13-24 represented roughly $26 \%$ of the new HIV

\footnotetext{
${ }^{a}$ Angela Bowen Williamson Professor of Epidemiology and Biostatistics and Nutrition, (exb11@case.edu), Departments of Epidemiology and Biostatistics and Nutrition, Prevention Research Center for Healthy Neighborhoods, Case Western Reserve University, School of Medicine, 4th Floor, BioEnterprise Building, 10900 Euclid Avenue, Cleveland, OH 44106-7069. bAssistant Dean for Interprofessional Education, (KTufts@odu.edu), College of Health Sciences, Old Dominion University, 2150 Health Sciences Building, Norfolk, VA 23529. 'Assistant Professor, (est2@case.edu), Department of Epidemiology and Biostatistics, Prevention Research Center for Healthy Neighborhoods, Case Western Reserve University, School of Medicine, 4th Floor, BioEnterprise Building, 10900 Euclid Avenue, Cleveland, Ohio 44106-7069.

dAssociate Vice-Provost for Research and Graduate Studies, Professor of Nursing, (laura.hayman@umb.edu), College of Nursing and Health Sciences, University of Massachusetts Boston, 100 Morrissey Blvd. Boston, MA 02125-3393.

e'Data Manager, (Idanosky@gmail.com), Survey Research Center, Institute for Social Research, University of Michigan, 426 Thompson Street, Ann Arbor, Ml $48104-2321$.

fVice President, Academic and Research, (llovegreen@nlc.bc.ca), Northern Lights College, 11401 8th Street, Dawson Creek, British Columbia, Canada, V1G 4G2.

Address correspondence to: Elaine A. Borawski, Angela Bowen Williamson Professor of Epidemiology and Biostatistics and Nutrition, (exb11@case.edu), Departments of Epidemiology and Biostatistics and Nutrition, Prevention Research Center for Healthy Neighborhoods, Case Western Reserve University, School of Medicine, 4th Floor, BioEnterprise Building, 10900 Euclid Avenue, Cleveland, OH 44106-7069.
}

The research on which this article is based was funded by grant R01-HD041364 from the National Institute of Child Health and Human Development. 
diagnoses in $2010,57 \%$ of which were among young African Americans. ${ }^{2}$ It is essential that all adolescents learn behaviors that can help them lower the risk of acquiring or transmitting HIV and other STIs.

Schools have long been considered the logical setting for the dissemination and acquisition of information about HIV and STIs, including prevention strategies. ${ }^{3}$ There is evidence to suggest that parents feel that their children, particularly their high school children, should learn this information and if not through their traditional health and science classes, from a medical or health professional. ${ }^{4,5}$ School nurses have always been useful in enhancing health protective behavior ${ }^{6}$ as well as providing one-on-one instruction and guidance to adolescents regarding their reproductive health. ${ }^{7}$ However, for the most part, school-based instruction on reproductive health and the prevention of disease (eg, STIs, HIV) has been carried out by health education and science teachers ${ }^{8}$ who have received varying levels of preparation to deliver such programming. ${ }^{9}$

The purpose of this study was to examine whether the effectiveness (ie, improved knowledge, selfefficacy, intentions, compared to a control group) of a well-established HIV/STI prevention curricula (Be Proud! Be Responsible! [BPBR]) would vary based on facilitator type (health education classroom teacher vs school nurse). These analyses are part of a larger replication study published previously, ${ }^{10}$ looking at the effectiveness of BPBR ${ }^{11,12}$ when taught within the high school health education curricula and compared to a control intervention similar in delivery and dosage.

\section{METHODS}

\section{Sample and Procedure}

The study population was comprised of all 9th and 10th grade students enrolled in mandatory health education classes in the 10 participating high schools $(\mathrm{N}=1576)$. Details of the consent and student assent procedures are outlined elsewhere. ${ }^{10}$ The rate of refusal by parents and students was $5.9 \% \quad(\mathrm{~N}=93)$ and $1.6 \%(\mathrm{~N}=26)$, respectively, and $6.3 \%(\mathrm{~N}=100)$ of students were unavailable (ie, no longer attending, inconsistent attendance, expulsion, or transferred) to complete the pretest prior to the start of the curriculum. This yielded a final baseline sample of 1357 students.

A detailed description of the methods used in the larger study has been published previously. ${ }^{10}$ Briefly, 5 pairs of high schools were recruited; each pair selected based on their location and similarity with regard to community socioeconomic status (\% poverty) and racial composition of the student body. Within each pair, schools were randomized using a 2stage, double-blinded randomization procedure, ${ }^{13}$ to receive either the BPBR curriculum or Get Connected!, a comparison curriculum developed by the Cleveland Health Museum, focused on general health and wellness. ${ }^{10}$ The curricula were taught in health classes either by the health education teacher or in $25 \%$ of the classes, by the school nurse, with school nurse-led classrooms also selected by randomization.

The BPBR curriculum consists of 6 modules of 50 minutes each that include a variety of developmentally appropriate teaching methods, such as group discussions, role model stories depicted in videos, interactive exercises, and role-playing. ${ }^{10,11,14}$ Details of the curriculum, specific modifications made, and details regarding the facilitator training are discussed elsewhere. ${ }^{10}$

The teacher and nurse facilitators for both curricula attended separate 2 -day ( 12 hours in total) training sessions. They were instructed on how to complete a detailed checklist for each session, including rating their command of the materials, their rapport with the students, the orderliness of the classroom, and the extent to which the material for each session was covered, while documenting any deviations from the original curriculum. In addition, each facilitator was observed at least once during the 6 curriculum sessions to assess their comfort level with the material and fidelity to the curriculum.

Data were collected from participating students using confidential self-administered questionnaires, administered at 4 time points: prior to the intervention (baseline), immediately following the intervention (within 2 weeks of final class session), 4 and 12 months following the intervention. Of the 1357 baseline students, 99\% completed the immediate post-test, $97 \%$ completed the 4-month follow-up, and $92 \%$ completed the 12-month follow-up survey. Rates of follow-up did not differ between the intervention and control groups.

\section{Measures}

The guiding theoretical framework for the intervention study posits that the intervention will influence sex-related behaviors both directly as well as indirectly through cognitive processes that are assumed to mediate behavioral change. ${ }^{12}$ Five categories of sex-related cognitive mediators were included in the study: knowledge, efficacy, participants' beliefs, perceived peer beliefs, and behavioral intentions. Sexual behavior was measured; however, because of the lack of significant intervention effects on adolescent sexual behavior in the main trial analyses, ${ }^{10}$ we limit the analyses here to the cognitive factors associated with behavioral change and the target of classroom instruction. Reported alpha reliabilities reflect internal consistency reliability of scaled measures.

Knowledge. Two domains of knowledge were assessed: condom knowledge (5 items; eg, "A condom 
should be completely unrolled before it is placed on the penis") and HIV/STI knowledge (7 questions; eg, "There's a good chance you'll get AIDS if you share a sink, shower or toilet seat with a person who has AIDS"). Correct responses for each domain were summed yielding scores ranging from 0 to 5 for condom knowledge and 0 to 7 for HIV/STI knowledge.

Intentions. Intention to have sexual intercourse was measured by 3 items; responses ranged from "Definitely likely" (5) to "Not at all likely" (1), with items for the scale summed and averaged. Two items assessed the expectation of having sex in the next 3 months and in the next year, and the third assessed the expectation of not having sex (being abstinent) until after high school (reversecoded); higher scores indicated higher intentions to engage in sex (alphas, 0.90-0.94 across study time points). Participants' intention to use a condom should they have sex was measured by a single item; higher scores indicated a greater intention of using a condom.

Efficacy. Three types of self-efficacy were measured; responses ranged from "Totally sure" (5) to "Totally unsure" (1), with items for each scale summed and averaged. Impulse control was measured using 2 items (eg, "How sure are you that you could keep from having sex until you feel ready?"); higher scores indicated students' greater confidence in their ability to resist unwanted sexual advances (correlations, 0.81-0.87). Condom negotiation skills were measured using 3 items (eg, "I can get my partner to use a condom even if he/she does not want to" ); higher scores indicated students' greater confidence in their ability to get a partner to agree to use a condom (alphas, 0.60-0.64). Condom technical skills were measured by 3 items (eg, "How sure are you that you could use a condom correctly or explain to your partner how to use a condom correctly"); higher scores showed students' greater confidence in their ability to correctly use a condom (alphas, 0.67-0.72).

Beliefs. Beliefs about 3 aspects of condom use were assessed; responses ranged from "Definitely yes" (5) to "Definitely no" (1), with items for each scale summed and averaged. The condom use belief scale comprised 3 items measuring students' perspective of the value and importance of using condoms (eg, "I believe condoms should always be used if a person my age has sex, even if the girl uses birth control"); higher scores indicated greater belief in the importance of condom use (alphas, 0.86-0.89). The condom use hedonistic scale consisted of three items measuring perceptions of whether condoms interfered with sexual enjoyment (eg, "Sex feels unnatural when a condom is used"); higher scores indicated a belief that condoms interfere (alphas, 0.55-0.61). The two items of the condom use prevention scale measured the belief that condoms prevent HIV and other sexually transmitted diseases (eg, "As long as I use a condom during sex, I know I will be safe from disease"); higher scores indicated greater belief in the protective quality of condoms (correlations, 0.42-0.64).

Perceived peer beliefs. Three items (summed and averaged) measured peer beliefs of the acceptability of sexual activity (eg, "Most of my friends believe it's okay for people my age to have sex with a steady boyfriend or girlfriend"). Responses ranged from "definitely yes" (5) to "definitely no" (1); higher scores indicated increased acceptability (alphas, 0.640.72). Perception of peers' beliefs regarding condom use was also measured by three items (eg, "Most of my friends believe condoms should always be used if a person my age has sex, even if the girl uses birth control"). Responses ranged from "definitely yes" (5) to "definitely no" (1); higher scores indicated stronger perceptions that peers believed in the importance of condom use (alphas, 0.85-0.87).

Descriptive characteristics. Age, sex, ethnicity (white, black, Hispanic, other), living arrangement (with 2 parents vs other), and parents' education (at least one parent having had some postsecondary education versus other) were assessed at baseline. An estimation of students' socioeconomic status was generated by using the proportion of households in their neighborhood that were at or below the federal poverty line, based on the 2000 US Census (range, $1-70 \%)$; this was done by linking the student's address to data for that specific census tract. Sexual experience at baseline was also assessed.

Curriculum fidelity, facilitator performance, and student assessment. Facilitators completed a checklist after each of the 6 class sessions. In addition to attendance, they recorded their command of the session materials, their perceived rapport with students, and how closely they followed the original curriculum as outlined in the training manual. They also recorded whether they completed each of the specific activities (25 in all) within the designated sessions (and if not, why), and if they were unable to complete an activity, whether they completed it at a later session. To assess the student perception of facilitator performance, students were queried at the immediate post-test as to how well facilitators presented the material and how comfortable they were in doing so; whether the curriculum challenged how students thought about their health; how comfortable students were with the activities and with potentially discussing a personal concern with their facilitator; the general classroom environment; and how seriously their peers regarded the curriculum. Each of the 7 student perception questions were measured with 5-point responses, ranging from 1 (lowest perception) to 5 (highest perception). 


\section{Statistical Analysis}

Bivariate statistics were used to assess whether facilitator characteristics, facilitator self-report of performance, and student perception of performance differed by facilitator type (school nurse or health education teacher). Continuous independent variables were compared using t-tests; Fisher's exact test was used to compare categorical variables. When comparing student perception of instructor performance, the analyses included the cluster option in SAS survey procedures, which adjusts the standard errors of estimates for intragroup correlations.

Previous analyses identified small but significant differences of participating students in the intervention and control groups in sex, ethnicity, neighborhood socioeconomic status, and session attendance. ${ }^{10}$ Therefore, to control for possible confounding in the analysis of the cognitive mediators, these variables, as well as participant age at baseline and baseline sexual experience (yes $=1$, no $=0$ ), were included as covariates in subsequent analyses.

To explore intervention effects by facilitator and report the findings in the most intuitive and straightforward method, we stratified the sample by facilitator type and used general linear modeling, with group membership (intervention $=1$, controls $=0$ ) as the fixed effect and covariates, including those mentioned above as well as baseline measure of the outcome variable; this approach produced adjusted group means or proportions for comparison.

While providing intuitive estimates, this analytic method does not accommodate for the complexity of the study design and randomization at the school level. To account for the additional variance anticipated due to the cluster-sampling design and the fact that observations obtained from students in the same school are likely to be correlated, we conducted general linear mixed modeling and included school in the model as a random effect. ${ }^{15}$ This method also incorporates hierarchical linear analysis techniques which allow school-level predictors, such as intervention group membership, to be accurately modeled as group-level (ie, school) covariates. ${ }^{16}$ Thus, analyses were conducted using the SAS software package (version 9.2 for Windows; SAS Institute Inc., Cary, NC, 2008) MIXED procedure to adjust for potential design effect of clustering at the school level.

Using SAS PROC MIXED, we fit models stratified by facilitator type to assess the relationship between intervention group membership and dependent variables (eg, cognitive mediators) while simultaneously adjusting for identified covariates, while also including school membership as a random effect. Owing to the intuitive presentation of the adjusted means and proportions of the general linear model, these estimates are presented, yet the statistical significance of differences between the intervention and control groups is based on the adjusted results from SAS PROC MIXED. Because of insignificant changes in behavior in the parent study, ${ }^{10}$ we did not examine the intervention effects on behavioral outcomes stratified by facilitator type.

\section{RESULTS}

The facilitators $(\mathrm{N}=27)$ were comprised of 6 school nurses ( 2 control, 4 intervention) and 21 health education classroom teachers ( 12 control, 9 intervention) from the 10 participating high schools. Demographic characteristics of the facilitators are shown in Table 1. The school nurses serving as facilitators were older than the classroom teachers ( 50.8 years vs 42.3 years, $p<.07)$ but were otherwise comparable in sex, race, education attained, and years within the school system. In general, most of the facilitators were female, held advanced degrees, and were white. All health education classroom teachers held degrees in either health education or physical education.

Table 2 describes facilitator self-report of classroom performance and curriculum fidelity. Facilitators reported high ratings of their command of the materials and rapport with the students; there were no significant differences reported between school nurses and health education teachers. School nurses reported completing a higher percentage of overall activities in their curriculum than the health education teachers $(81.0 \%$ vs $70.6 \%, \mathrm{p}<.09)$; however, these differences were related to the number of activities, such as the

Table 1. Demographic Characteristics of Facilitators

\begin{tabular}{lccc}
\hline & $\begin{array}{c}\text { School } \\
\text { Nurse } \\
(\mathbf{N}=\mathbf{6})\end{array}$ & $\begin{array}{c}\text { Classroom } \\
\text { Teacher } \\
\mathbf{( N = 2 1 )}\end{array}$ & $\mathbf{p}$ \\
\hline Age (years) & 50.8 & 42.3 & .07 \\
Sex (\% female) & 100.0 & 61.9 & .14 \\
Ethnicity (\% nonwhite) & 33.3 & 40.0 & .98 \\
Education attained (\% with & 66.7 & 75.0 & .92 \\
$\quad$ master's degree or higher) & & & \\
Years with school system & 13.8 & 16.9 & .48 \\
\hline
\end{tabular}

Table 2. Facilitator's Self-Report of Classroom Performance and Curriculum Fidelity

\begin{tabular}{lccc}
\hline & $\begin{array}{c}\text { School } \\
\text { Nurse } \\
(\mathbf{N}=6)\end{array}$ & $\begin{array}{c}\text { Classroom } \\
\text { Teacher } \\
(\mathbf{N}=\mathbf{2 1})\end{array}$ & $\mathbf{p}$ \\
\hline $\begin{array}{l}\text { Command of materials }(1=\text { low, } \\
\quad 10=\text { high) }\end{array}$ & 8.3 & 8.8 & .20 \\
$\begin{array}{l}\text { Rapport with students }(1=\text { low, } \\
10=\text { high) }\end{array}$ & 8.0 & 8.5 & .37 \\
$\begin{array}{l}\text { Percent of activities completed } \\
\text { Fidelity to original curriculum }(1=\text { not } \\
\text { at all close, 4= exactly) }\end{array}$ & 81.0 & 70.6 & .09 \\
\hline
\end{tabular}


Table 3. Students' Perceptions of Facilitators' Performance

\begin{tabular}{lccc}
\hline & $\begin{array}{c}\text { School } \\
\text { Nurse } \\
(\mathbf{N}=\mathbf{2 4 0})\end{array}$ & $\begin{array}{c}\text { Classroom } \\
\text { Teachers } \\
(\mathbf{N}=\mathbf{1 1 1 7})\end{array}$ & $\mathbf{p}$ \\
\hline $\begin{array}{l}\text { How well did the instructor } \\
\text { present the materials? }\end{array}$ & 3.01 & 3.19 & .058 \\
$\begin{array}{l}\text { How comfortable was the } \\
\text { instructor in presenting the } \\
\text { materials? }\end{array}$ & 3.22 & 3.43 & .006 \\
$\begin{array}{l}\text { How much did the curriculum } \\
\text { challenge the way you think } \\
\text { about your health? }\end{array}$ & 2.71 & 2.92 & .016 \\
$\begin{array}{l}\text { How comfortable were you } \\
\text { doing the activities in the } \\
\text { curriculum? }\end{array}$ & 3.10 & 3.07 & .647 \\
$\begin{array}{l}\text { How comfortable would you be } \\
\text { to discuss a personal concern } \\
\text { with this instructor? }\end{array}$ & 2.44 & 2.38 & .456 \\
$\begin{array}{l}\text { How seriously did the students } \\
\text { in your class take the } \\
\text { materials presented? }\end{array}$ & 2.95 & 3.26 & .075 \\
$\begin{array}{l}\text { In general, how would you } \\
\text { describe the classroom } \\
\text { environment during the } \\
\text { classes? }\end{array}$ & 3.26 & 3.66 & \\
\hline
\end{tabular}

number of role-plays, within a topic area rather than differences in topic areas covered. Both school nurses and health education classroom teachers reported a high degree of fidelity to the original curriculum.

Students' perception of facilitator performance is shown in Table 3. After adjusting for classroom level clustering, students gave higher ratings to the classroom teacher facilitators than to the school nurse facilitators in the areas of how comfortable the facilitator was with the materials $(p<.006)$ and the extent to which the curriculum challenged how students thought about their health $(p<.02)$. Although not significant, students also reported that their peers took the materials more seriously when delivered by the health education classroom teachers $(p<.08)$ and perceived the classroom environment of classroom teachers to be more orderly than with the school nurses $(p<.07)$.

Table 4 summarizes the impact of the intervention curriculum on cognitive mediators reported at immediate post-test, 4-month post-test, and 12-month post-test, stratified by school nurses and classroom heath education teachers. Compared to controls, intervention students reported significant increases in their HIV/STI and condom use knowledge immediately following the intervention, regardless of whether they were taught by a classroom teacher or school nurses. However, only those taught by the health education teacher retained the condom use knowledge beyond the immediate post-test. With regard to intentions, students taught by school nurses reported a change in their intentions to use a condom; however, this was only found at the immediate post-test and 4-month post-test. Students taught by health education teachers reported no changes in intentions.

In reporting self-efficacy around impulse control, condom negotiation, and condom technical skills, all students receiving the intervention showed a significant increase in their condom technical skills and condom negotiation skills immediately following the intervention; however, this effect was diminished by 4 months for students who were taught by the classroom teacher, but remained strong l year later for those taught by the school nurse. Similarly, intervention students taught by a school nurse at 12 months reported greater impulse control efficacy than the controls, while students taught by health education teachers reported no differences.

Compared to controls, intervention students taught by school nurses reported significant increases in their condom use beliefs at 4 months; significant increases in condom use-hedonistic beliefs and condom use-prevention beliefs at immediate posttest were sustained at 12 months and 4 months, respectively. In contrast, compared to controls, students taught by health education teachers reported an increase in condom use-prevention beliefs at immediate post-test only and a significant change in the undesired direction at 4 months for condom usehedonistic beliefs. There were no reported differences in reported peer beliefs.

\section{DISCUSSION}

Schools are a logical setting for disseminating information about HIV/STI prevention and risk reduction behaviors to adolescents, ${ }^{5,8,17,18}$ and most often this information is provided as part of the regular science and/or health education curricula. The results of this study suggest that while classroom health education teachers may be skilled at imparting knowledge, they may be less effective with instruction involving skills aimed at reducing risky sexual behaviors. Students reported more positive views of health education teachers' performance with regard to the presentation of materials, the comfort level of the facilitator, and the degree to which the curriculum challenged how students thought about health. However, students who participated in sessions that were taught by school nurses were more likely to report significant and sustainable changes in a broad range of sex-related cognitive mediators including selfefficacy, condom-related beliefs, and peer behavior beliefs while those taught by health education teachers reported long-term impact on condom knowledge only. While previous research has indicated that teens' perceptions of facilitators contributes to program outcomes, ${ }^{19}$ our results are consistent with previous research by Akpabio et $\mathrm{al}^{20}$ who found that attitudes 


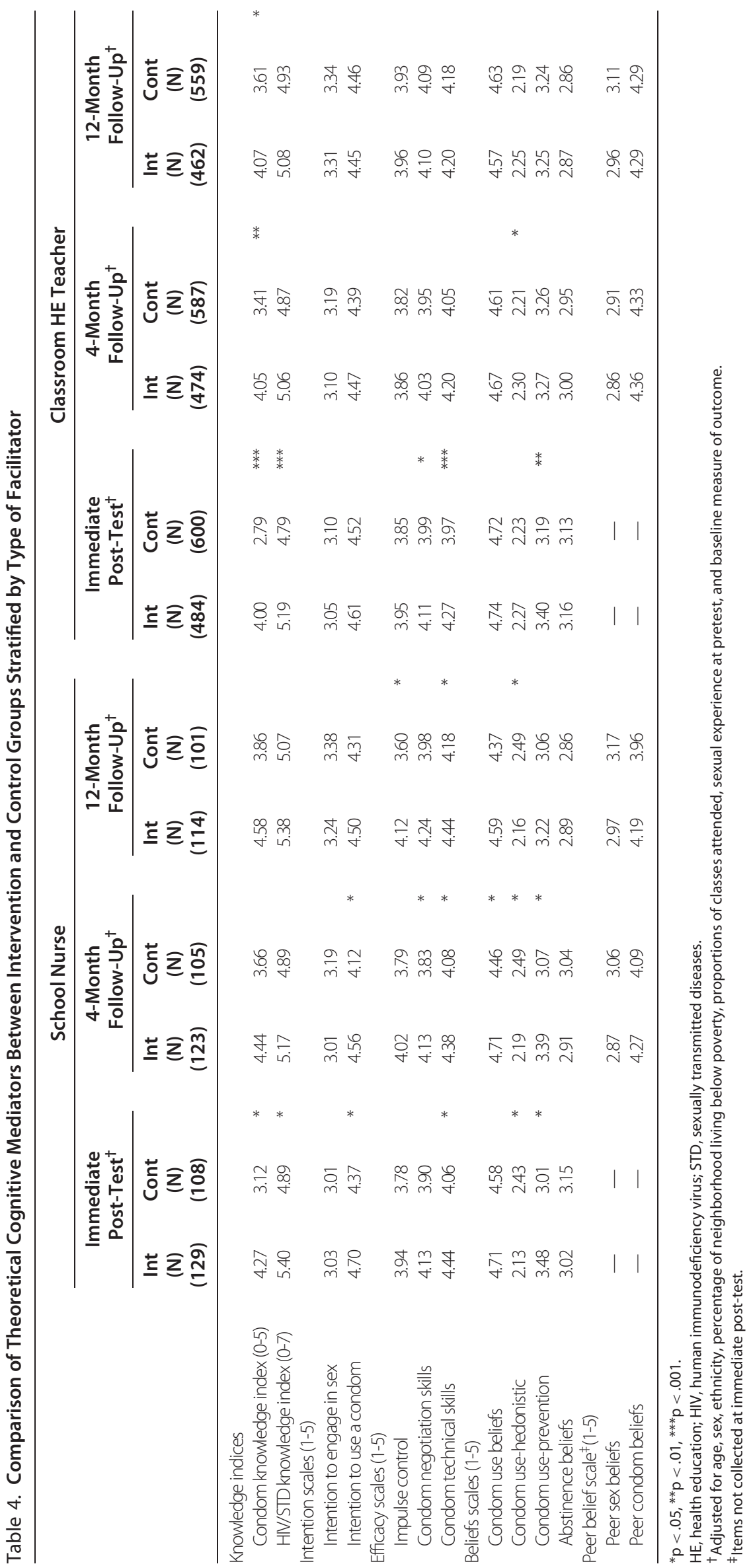


toward HIV preventative measures were most potent when given by nurses.

One possible explanation for these differences may be that nurses carried out a higher percentage of the required curricular activities. Although this trend did not reach statistical significance, the school nurses reported higher degrees of fidelity to the original curriculum. Whereas we did not anticipate this finding, the fact that adolescent and school nurse interactions regarding reproductive health issues usually occur one on one and not in a classroom may account for the school nurses' higher degree of fidelity. School nurses may have felt that in order to be more effective teachers they needed more preparation or that they needed to keep closer to the prescribed schedule of activities. Also, the experienced health education teachers were more likely to report via fidelity checklists that they dropped activities that they viewed as redundant (eg, carrying out 2 of the 4 role play activities on the same topic). Both scenarios could have led to school nurses more closely adhering to the curriculum than the health education teachers.

Another possible explanation is that as a profession, nurses have extensive experience talking to people about sensitive topics. Nurses have been rated as more sensitive interviewers ${ }^{21}$ and are expected to discuss a broad spectrum of reproductive health issues with individuals of all ages and backgrounds, including HIV/AIDS, sexual pleasure, and condom use. These expectations begin very early during a nurses' formal education with nurses in their first year of training being expected to become competent communicators on a wide range of sensitive topics. Being skilled at conducting sensitive discussions may result in nurses being more likely to address salient points, even if they are sensitive. School nurses in this study likely had discussed importance of using condoms, negotiation of condom use, how to control sexual impulses, and the mechanics of using a condom with numerous adolescents during one-on-one encounters. Thus, they were able to effectively apply these experiences to the classroom setting. In contrast, the training and role of classroom health education teachers with regard to teaching reproductive health is more varied and focuses more on the transfer of information than skill development, such as how HIV is transmitted versus how to correctly use a condom. ${ }^{9}$ A number of the teacher facilitators in this study split their time between teaching health, physical education, and coaching, thereby potentially reducing the opportunity and experience of discussing explicit reproductive health issues with adolescents, such as how to use a condom correctly. ${ }^{9}$

School nurses are also likely to have more experience with facilitating the acquisition of technical skills; teaching individuals to perform technical tasks ranging from self-injection of medication to colostomy care. $^{22,23}$ Nurses are recognized for their expertise in building self-efficacy and self-competence in patient encounters and interactions with individuals across healthcare and community-based settings. ${ }^{24}$ Thus, the nurses' skill in this area may have accounted for the students reported continued confidence in their ability to use a condom (condom technical skills) at 12 months while the confidence of students who were taught by health education teachers had diminished well before that time.

There were several limitations with this study. Because of the limited time of the school nurses, only $25 \%$ of the classes could be randomly assigned to school nurses, reducing the sample size and power to detect smaller intervention effects. For example, we lacked the sample size to stratify further the analyses by fidelity (percentage or type of activities covered) to determine whether the differences can be explained by dosage or specific content that was covered. Second, the study was limited to one geographic location where school nurse training and duties within schools may be unique, and thus, may not be generalizable to all school districts. ${ }^{25}$ Last, the sample size of teacher-led classrooms was not large enough to determine whether the training and education (physical education vs health education) of the health education teacher or primary role (classroom teacher, coach) played a role in the results.

\section{IMPLICATIONS FOR SCHOOL HEALTH}

Whereas involving school nurses in classroom reproductive health education may be the ideal recommendation of this study, it may not be realistic for most school districts today to commit school nurse time to the classroom due to the increasing student to nurse ratio and growing fiscal constraints. However, the results do suggest that involving a school nurse or another health professional that students are familiar and comfortable with would greatly enhance specific reproductive health teachings, especially those associated with skill building and reducing risky sexual health behaviors. It is unclear from our study what role familiarity with the school nurse played with the study outcomes and thus cannot conclude that similar outcomes would result from engaging an unfamiliar health professional. At the least, health education teachers should not bear the sole responsibility of teaching adolescents about healthy sexual behaviors; rather it should be conducted in a more comprehensive manner that involves classroom instruction, opportunities for guided role playing, and perhaps one-on-one engagement with a health professional, perhaps through a school clinic. Last, this study does suggest that heath education teachers do a good job with conveying knowledge in a compelling way that encourages long-term retention 
of knowledge. However, it also suggests that health education teachers may greatly benefit from additional training in methods for teaching sexual risk reduction among adolescents.

In summary, this study highlights the value of both health education teachers and school nurses in conveying reproductive health information to high school students. It also suggests that to be the most effective (ie, influencing risk behaviors), schools may wish to involve school nurses in teaching adolescents the technical and interpersonal (eg, negotiation) skills needed to protect themselves from high-risk sexual behavior.

\section{Human Subjects Approval Statement}

This study was approved by the Institutional Review Board of Case Western Reserve University.

\section{REFERENCES}

1. Centers for Disease Control and Prevention (CDC). Sexually Transmitted Disease Surveillance. Atlanta, GA: CDC; $2011: 2011$.

2. Centers for Disease Control and Prevention (CDC). HIVIAIDS Among Youth. Atlanta, GA: CDC; March 2014. Available at: http://www.cdc.gov/hiv/risk/age/youth/index.html?s_cid= tw_std0141316. Accessed December 27, 2014.

3. Kirby D. The impact of schools and school programs upon adolescent sexual behavior. J Sex Res. 2002;39(1):27-33.

4. Ito KE, Gizlice Z, Owen-O'Dowd J, Foust E, Leone PA, Miller WC. Parent opinion of sexuality education in a state with mandated abstinence education: does policy match parental preference? J Adolesc Health. 2006;39(5):634-641.

5. Lloyd SW, Ferguson YO, Corbie-Smith G, et al. The role of public schools in HIV prevention: perspectives from African Americans in the rural south. AIDS Educ Prev. 2012;24(1):41-53.

6. Barbara M. Teaching in Health. Philadelphia, PA: Mosby; 1996.

7. Barnes M, Walsh A, Courtney M, Dowd T. School based youth health nurses' role in assisting young people access health services in provincial, rural and remote areas of Queensland. Aust Rural Remote Health. 2004;4(4):279.

8. Wiefferink CH, Poelman J, Linthorst M, Vanwesenbeeck I, Van Wijngaarden JCM, Paulussen TGW. Outcomes of a systematically designed strategy for the implementation of sex education in Dutch secondary schools. Health Educ Res. 2005;20(3):323-333.

9. Herr S, Telliohann S, Price J, Dake J, Stone G. High school health-education teachers' perceptions and practices related to teaching HIV prevention. J Sch Health. 2012;82(11):514-521.
10. Borawski EA, Trapl ES, Adams-Tufts K, Hayman LL, Goodwin MA, Lovegreen LD. Taking Be Proud! Be Responsible! to the suburbs: a replication study. Perspect Sex Reprod Health. 2009;41(1):12-22.

11. Jemmott L, Jemmott J, McCaffree K. Be Proud! Be Responsible! Strategies to Empower Youth to Reduce Their Risk for AIDS. Curriculum Manual. New York, NY: Select Media; 1996.

12. Jemmott JB, Jemmott LS, Fong GT. Reductions in HIV riskassociated sexual behaviors among black male adolescents: effects of an AIDS prevention intervention. Am J Public Health. 1992;82(2):372-377.

13. Green S, Corle D, Gail M, et al. Interplay between design and analysis for behavioral intervention trials with community as the unit of randomization. Am J Epidemiol. 1995;142(6):587593.

14. Jemmott JB, Jemmott LS, Fong GT. Abstinence and safer sex HIV risk-reduction interventions for African American adolescents: a randomized controlled trial. JAMA. 1998;279(19):15291536.

15. Murray DM. Design and Analysis of Group-Randomized Trials. New York, NY: Oxford University Press; 1998.

16. Singer JD, Using SAS. PROC MIXED to fit multilevel models, hierarchical models, and individual growth models. J Educ Behav Stat. 1998;23(4):323-355.

17. Joronen K, Rankin SH, Astedt-Kurki P. School-based drama interventions in health promotion for children and adolescents: systematic review. J Adv Nurs. 2008;63(2):116-131.

18. Kirby DB, Laris BA, Rolleri LA. Sex and HIV education programs: their impact on sexual behaviors of young people throughout the world. J Adolesc Health. 2007;40(3):206-217.

19. Jarrett T, Horn K, Zhang J. Teen perceptions of facilitator characteristics in a school-based smoking cessation program. $J$ Sch Health. 2009;79(7):297-303.

20. Akpabio II, Asuzu MC, Fajemilehin BR, Ofi AB. Effects of school health nursing education interventions on HIV/AIDSrelated attitudes of students in Akwa Ibom State. Nigeria $J$ Adolesc Health. 2009;44(2):118-123.

21. Jonasson L-L, Liss P-E, Westerlind B, Berterö C. Ethical values in caring encounters on a geriatric ward from the next of kin's perspective: an interview study. Int J Nurs Pract. 2010;16(1): 20-26.

22. Gordon S. What do nurses really do? Top Adv Pract Nurs eJournal. 2006;6(1). Available at: http://www.medscape. com/viewarticle/520714_2. Accessed September 25, 2014.

23. Johnston JH. The preparation of child health nurses in sexual health education: an exploratory study. Nurse Educ Today. 2009;29(8):845-849.

24. Gordon SC, Barry CD. Delegation guided by school nursing values: comprehensive knowledge, trust, and empowerment. $J$ Sch Nurs. 2009;25(5):352-360.

25. Nwabuzor O. Legislative: shortage of nurses: the school nursing experience. Online J Issues Nurs. 2007;12(2):10. 